\title{
NORM INEQUALITIES EQUIVALENT TO HEINZ INEQUALITY
}

\author{
JUN ICHI FUJII, MASATOSHI FUJII, TAKAYUKI FURUTA, AND RITSUO NAKAMOTO
}

(Communicated by Palle E. T. Jorgensen)

Dedicated to Professor Tsuyoshi Ando on his sixtieth birthday

\begin{abstract}
We investigate several norm inequalities equivalent to the Heinz inequality and discuss the equivalence relations among these norm inequalities. Here we shall show an elementary and simplified proof to the famous Heinz inequality.
\end{abstract}

\section{INTRODUCTION}

A capital letter denotes a bounded linear operator on a Hilbert space. In 1951 Heinz [7] proved a series of very useful norm inequalities that are closely related to the Cordes inequality [3,4] and the Furuta inequality [5]. In particular, inequality (1) in Theorem 1 is said to be the Heinz inequality, which is one of the most essential inequalities in operator theory. Its original proof in [7], however, is based on the complex analysis theory and is somewhat complicated. Here we shall show an elementary and simplified proof of the famous Heinz inequality.

We are inspired by inequality (4) in Theorem 1 by Corach, Porta, and Recht [2], which is a key factor in their study of differential geometry; we received our initiation and the means to give an elementary proof to the Heinz inequality by reading and scrutinizing [2]. Also we cannot but emphasize the importance of [8] by McIntosh, since we think that it is not widely known.

In this paper, we shall investigate several norm inequalities equivalent to Heinz inequality and also, by elementary calculations, we shall show that they are mutually equivalent. Consequently, we can give an elementary proof to the Heinz inequality.

\section{EQUIVALENCE AMONG NORM INEQUALITIES}

First we show the following result.

Theorem 1. The following norm inequalities (1), (2), (3), (4), (5), and (6) hold and follow from each other.

$$
\left\|S_{1} Q+Q S_{2}\right\| \geq\left\|S_{1}^{\alpha} Q S_{2}^{1-\alpha}+S_{1}^{1-\alpha} Q S_{2}^{\alpha}\right\|,
$$

Received by the editors April 21, 1991 and, in revised form, October 28, 1991.

1991 Mathematics Subject Classification. Primary 47A30, 47B15.

Key words and phrases. Heinz inequality, positive operator. 
where $S_{1}$ and $S_{2}$ are positive operators and $1 \geq \alpha \geq 0$;

$$
\begin{gathered}
\left\|P^{*} P Q+Q R R^{*}\right\| \geq 2\|P Q R\|, \\
\left\|S T R^{-1}+S^{-1} T R\right\| \geq 2\|T\|,
\end{gathered}
$$

where $S$ and $R$ are selfadjoint and invertible operators;

$$
\left\|S T S^{-1}+S^{-1} T S\right\| \geq 2\|T\|,
$$

where $S$ is a selfadjoint and invertible operator;

$$
\left\|S T S^{-1}+S^{-1} T S\right\| \geq 2\|T\|,
$$

where $S$ is a selfadjoint and invertible operator and $T$ is a selfadjoint operator; and

$$
\left\|A^{2 m+n} T B^{-n}+A^{-n} T B^{2 m+n}\right\| \geq\left\|A^{2 m} T+T B^{2 m}\right\|,
$$

where $A$ and $B$ are selfadjoint and invertible operators and $m, n$ are both nonnegative integers.

Inequality (1) is due to Heinz [7] and (2) is obtained by McIntosh [8] to prove (1); its proof is very ingenious. In fact, using (2), he proved that $f(\alpha)=$ $\left\|S_{1}^{\alpha} Q S_{2}^{1-\alpha}+S_{1}^{1-\alpha} Q S_{2}^{\alpha}\right\|$ is convex on $[0,1]$. (3) is a generalization of inequality (4), which was recently proved by Gorach, Porta, and Recht [3]. (5) is nothing but a special case of (4). (6) can be considered as an extension of (3).

Proof of Theorem 1. We shall show the following implications: $(1) \rightarrow(6) \rightarrow$ $(5) \rightarrow(4) \rightarrow(3) \rightarrow(2) \rightarrow(1)$.

$(1) \rightarrow(6)$. By choosing, $\alpha=(2 m+n)(2 m+2 n)^{-1}, S_{1}=A^{2 m+2 n}, S_{2}=$ $B^{2 m+2 n}$, and $Q=A^{-n} T B^{-n}$ in (1), we obtain (6) by (1).

$(6) \rightarrow(5)$. Obvious.

$(5) \rightarrow(4)$. As $\left(\begin{array}{cc}0 & T \\ T^{*} & 0\end{array}\right)$ is selfadjoint, we have

$$
\begin{aligned}
& \left\|\left(\begin{array}{ll}
S & 0 \\
0 & S
\end{array}\right)\left(\begin{array}{cc}
0 & T \\
T^{*} & 0
\end{array}\right)\left(\begin{array}{cc}
S & 0 \\
0 & S
\end{array}\right)^{-1}+\left(\begin{array}{cc}
S & 0 \\
0 & S
\end{array}\right)^{-1}\left(\begin{array}{cc}
0 & T \\
T^{*} & 0
\end{array}\right)\left(\begin{array}{cc}
S & 0 \\
0 & S
\end{array}\right)\right\| \\
& \quad \geq 2\left\|\left(\begin{array}{cc}
0 & T \\
T^{*} & 0
\end{array}\right)\right\|,
\end{aligned}
$$

so that we obtain

$$
\left\|\left(\begin{array}{cc}
0 & S T S^{-1}+S^{-1} T S \\
S T^{*} S^{-1}+S^{-1} T^{*} S & 0
\end{array}\right)\right\| \geq 2\left\|\left(\begin{array}{cc}
0 & T \\
T^{*} & 0
\end{array}\right)\right\| .
$$

Hence it follows that $\left\|S T S^{-1}+S^{-1} T S\right\| \geq 2\|T\|$ since $\left\|\left(\begin{array}{cc}0 & X \\ X^{*} & 0\end{array}\right)\right\|=\|X\|$.

$(4) \rightarrow(3)$. We have only to use Berberian's magic in [1] as follows:

$$
\begin{aligned}
& \left\|\left(\begin{array}{ll}
S & 0 \\
0 & R
\end{array}\right)\left(\begin{array}{ll}
0 & T \\
0 & 0
\end{array}\right)\left(\begin{array}{cc}
S & 0 \\
0 & R
\end{array}\right)^{-1}+\left(\begin{array}{cc}
S & 0 \\
0 & R
\end{array}\right)^{-1}\left(\begin{array}{ll}
0 & T \\
0 & 0
\end{array}\right)\left(\begin{array}{ll}
S & 0 \\
0 & R
\end{array}\right)\right\| \\
& \quad \geq 2\left\|\left(\begin{array}{ll}
0 & T \\
0 & 0
\end{array}\right)\right\|,
\end{aligned}
$$

so that we have

$$
\left\|\left(\begin{array}{cc}
0 & S T R^{-1}+S^{-1} T R \\
0 & 0
\end{array}\right)\right\|=\left\|S T R^{-1}+S^{-1} T R\right\| \geq 2\|T\| .
$$


$(3) \rightarrow(2)$. We may assume that $P^{*} P$ and $R R^{*}$ are both invertible. Then we get

$$
\begin{aligned}
\left\|P^{*} P Q+Q R R^{*}\right\| & =\left\||P||P| Q\left|R^{*}\right|\left|R^{*}\right|^{-1}+|P|^{-1}|P| Q\left|R^{*}\right|\left|R^{*}\right|\right\| \\
& \geq 2\left\||P| Q\left|R^{*}\right|\right\|=2\|P Q R\| .
\end{aligned}
$$

$(2) \rightarrow(1)$. An elegant proof of this implication is shown by McIntosh in [8], which is quite similar to one by Pedersen [9]. For the sake of completeness, we cite its proof. We first note that (1) holds true for $\alpha=0,1$. Suppose that $0 \leq \mu=\alpha-p<\alpha<\alpha+p=\lambda \leq 1$ and that (1) holds true for $\mu$ and $\lambda$. Then it follows from (2) that

$$
\begin{aligned}
f(\alpha) & =\left\|S_{1}^{\alpha} Q S_{2}^{1-\alpha}+S_{1}^{1-\alpha} Q S_{2}^{\alpha}\right\| \\
& =\left\|S_{1}^{p}\left(S_{1}^{\mu} Q S_{2}^{1-\lambda}+S_{1}^{1-\lambda} Q S_{2}^{\mu}\right) S_{2}^{p}\right\| \\
& \leq\left\|S_{1}^{2 p}\left(S_{1}^{\mu} Q S_{2}^{1-\lambda}+S_{1}^{1-\lambda} Q S_{2}^{\mu}\right)+\left(S_{1}^{\mu} Q S_{2}^{1-\lambda}+S_{1}^{1-\lambda} Q S_{2}^{\mu}\right) S_{2}^{2 p}\right\| / 2 \\
& \leq\left\|S_{1}^{\lambda} Q S_{2}^{1-\lambda}+S_{1}^{1-\lambda} Q S_{2}^{\lambda}\right\| / 2+\left\|S_{1}^{\mu} Q S_{2}^{1-\mu}+S_{1}^{1-\mu} Q S_{2}^{\mu}\right\| / 2,
\end{aligned}
$$

that is,

$$
f(\alpha) \leq(f(\alpha+p)+f(\alpha-p)) / 2 .
$$

Now $S_{1}^{\alpha}$ and $S_{2}^{\alpha}$ depend continuously on $\alpha \in(0,1]$ in the operator norm topology, and so the function $f(\alpha)$ is continuous on $(0,1)$. It follows from this and the above inequality that $f(\alpha)$ is convex on $[0,1]$. Moreover, since $f(\alpha)$ is symmetric about $\alpha=\frac{1}{2}$, it is decreasing on $\left[0, \frac{1}{2}\right]$, so that we obtain the desired inequality (1).

\section{A Simplified PRoOf to the Heinz Inequality Via Theorem 1}

The original proof of the Heinz inequality in [7] is based on the complex analysis theory and it requires complicated calculations. Here we give a simplified proof to this inequality.

Proof of (5). Let $\lambda \neq 0, \lambda \in \sigma(T)$. Then $\lambda \in \sigma\left(S T S^{-1}\right) \subset \bar{W}\left(S T S^{-1}\right)$, and so, since $\lambda$ is real, $2 \lambda \in 2 \operatorname{Re} \bar{W}\left(S T S^{-1}\right)=\bar{W}\left(S T S^{-1}+S^{-1} T S\right)$. Therefore $2\|T\|=2 r(T) \leq\left\|S T S^{-1}+S^{-1} T S\right\|$, so that the proof of (5) is complete.

Finally we obtain the Heinz inequality (1) by Theorem 1.

Remark 1. Using inequality (3), we have the following somewhat interesting inequality:

$$
\left\|S T S+S^{-1} T S^{-1}\right\| \geq 2\|T\|,
$$

where $S$ is a selfadjoint invertible operator.

Remark 2. Corach, Porta, and Recht [2] remarked that if either $T=T^{*}$ or $T=-T^{*}$, then (4) implies

$$
\left\|S T S^{-1}\right\| \geq\|T\| \text {. }
$$

We would like to remark that (7) can be derived from Rota's theorem, cf. [6].

\section{ACKNOWLEDGMENT}

We would like to express our thanks to Dr. Y. Kato that he has kindly given a copy of [8] to the fourth author.

Also we would like to express our thanks to the referee for useful comments. 


\section{REFERENCES}

1. S. K. Berberian, Note on a theorem of Fuglede and Putnam, Proc. Amer. Math. Soc. 10 (1959), 175-182.

2. G. Corach, H. Porta, and L. Recht, An operator inequality, Linear Algebra Appl. 142 (1990), 153-158.

3. J. I. Fujii and M. Fujii, A norm inequality for operator monotone functions, Math. Japon. 35 (1990), 249-252.

4. T. Furuta, Norm inequalities equivalent to Löwner-Heinz theorem, Rev. Math. Phys. 1 (1989), 135-137.

5. $\ldots, A \geq B \geq 0$ assures $\left(B^{r} A^{p} B^{r}\right)^{1 / q} \geq B^{(p+2 r) / q}$ for $r \geq 0, p \geq 0, q \geq 1$ with $(1+2 r) q \geq p+2 r$, Proc. Amer. Math. Soc. 101 (1987), 85-88.

6. T. Furuta and R. Nakamoto, On the numerical range of an operator, Proc. Japan Acad. 47 (1971), 279-284.

7. E. Heinz, Beiträge zur Störungstheorie der Spectralzerlegung, Math. Ann. 123 (1951), 415438.

8. A. McIntosh, Heinz inequalities and perturbation of spectral families, Macquarie Math. Reports, 1979.

9. G. K. Pedersen, Some operator monotone functions, Proc. Amer. Math. Soc. 36 (1972), 309-310.

Department of Arts and Sciences, Osaka Kyoiku University, Kashiwara, Osaka 582, JAPAN

Department of Mathematics, Osaka Kyolku University, Tennoji, Osaka 543, Japan

Department of Applied Mathematics, faculty of Science, Science University of Tokyo, Kagurazaka, Shinjuku 162, Tokyo, Japan

Faculy of Engineering, Ibaraki University, Hitachi, Ibaraki 316, Japan 